\title{
Ácido tranexámico en la medicina prehospitalaria en México
}

\author{
Ricardo A. Pingarrón-Ríos ${ }^{1 *}$, Iván Jiménez-Flores², Laura P. Soriano-Palomino ${ }^{3}$ y \\ Ma. Fernanda Villa-Domínguez ${ }^{4}$
}

${ }^{1}$ Servicio de Anestesiología, Fundación Clínica Médica Sur, Ciudad de México; ${ }^{2}$ Servicio de Urgencias, Fundación Clínica Médica Sur, Ciudad de México; ${ }^{3}$ Área de socorros, Cruz Roja Mexicana, Edo. Méx.; ${ }^{4}$ Urgencias médicas nivel intermedio, Cruz Roja Mexicana, Ciudad de México. México

\section{Resumen}

El ácido tranexámico se administra usualmente a escala mundial en las hemorragias masivas. Recientemente se describe su aplicación en distintas especialidades de manera más frecuente, incluyendo la medicina prehospitalaria. En México no se utiliza este medicamento de manera habitual en dicho ámbito pese a que el trauma y la hemorragia son algunas de las principales causas de muerte en la población joven. El propósito del presente artículo es fundamentar con la evidencia existente por qué se deberá considerar el uso de este medicamento en México de forma prehospitalaria.

Palabras clave: Tranexámico. Ácido. Prehospitalario. Urgencias. México. Hemorragia.

\section{Tranexamic acid in prehospital medicine in Mexico}

\begin{abstract}
Tranexamic acid is used on a worldwide scale; it is usually administered in massive hemorrhages. Recently, its application in different specialties has been described more frequently, including prehospital medicine. In Mexico, this medication is not used regularly in that subspecialty despite the fact that trauma and hemorrhage are some of the main causes of death among the young population. The purpose of this article is to substantiate with the existing evidence, why the use of this drug in Mexico must be considered for prehospital hemorrhage management.
\end{abstract}

Key words: Tranexamic. Acid. Prehospital. Emergency. Mexico. Hemorrhage.

\section{Introducción}

Una de las principales causas de muerte en la población mexicana joven, es decir, de 15 a 25 años, es la hemorragia asociada al trauma; también es una de las causas más importantes de muerte materna en México y en el ámbito internacional ${ }^{1-3}$.

La razón de mortalidad materna calculada en el año 2015 fue de 31.7 defunciones por cada 100,000 nacimientos estimados, de la cual el $22.3 \%$ está relacionada con hemorragia obstétrica 4 .

Descubierto en 1962 por la Dra. Utako Okamoto ${ }^{5}$, el ácido tranexámico (ATX) es un medicamento antifibrinolítico que ha atraído considerable atención de investigadores, médicos y personal prehospitalario en la última década. El ATX es un inhibidor competitivo del sitio de unión a lisina del plasminógeno ${ }^{6,7}$. En este

\section{Correspondencia:}

*Ricardo A. Pingarrón-Ríos

E-mail: rapingarronr@gmail.com
Fecha de recepción: 28-06-2019

Fecha de aceptación: 17-02-2021

DOI: 10.24875/REIE.19000047
Disponible en internet: 16-04-2021

Rev Educ Investig Emer. 2021;3(1):9-14 www.medicinadeemergencias.com

2604-6520 @ 2021 Sociedad Mexicana de Medicina de Emergencias, AC. Publicado por Permanyer México SA de CV. Este es un artículo open access bajo la licencia CC BY-NC-ND (http://creativecommons.org/licenses/by-nc-nd/4.0/). 
contexto, cuando se forma un coágulo, se inicia naturalmente un proceso de degradación de la fibrina, llamado fibrinólisis, considerado el principal modulador en la hemostasia. Este proceso, en trauma y hemorragias, se acelera debido a los altos niveles de factores fibrinolíticos en la circulación. Esto contribuye a una serie de reacciones que alteran los procesos de coagulación del cuerpo y contribuyen a la muerte. Esta sucesión de eventos se denomina hiperfibrinólisis ${ }^{8}$ y es uno de los factores de coagulopatía en la hemorragia crítica (Fig. 1) $)^{3,6,9}$.

El ATX previene tal reacción, ya que impide la activación del plasminógeno a la enzima plasmina, cuya función es romper los coágulos ya formados. Así, el proceso normal de la fibrinólisis se ve obstaculizado y se evita que la degradación en la cascada de la coagulación se desarrolle. Esto es favorable en el contexto de una hemorragia masiva, debido a que los coágulos seguirán siendo viables y provocarán hemostasia ${ }^{10}$.

Mundialmente, este medicamento se usa en las hemorragias masivas. Se han descrito distintas aplicaciones de su uso, entre las cuales se incluyen: hemorragia gastrointestinal ${ }^{11}$, artroplastia de rodilla ${ }^{12}$, derivación de arterias coronarias ${ }^{13}$, odontología ${ }^{14}$, trauma en pediatría ${ }^{15}$ y hemorragia posparto ${ }^{16}$, entre otras. Sobre esta última existe el Consenso multidisciplinario para el manejo de la hemorragia obstétrica en el perioperatorio ${ }^{17}$, donde entre otras recomendaciones se sugiere que el manejo prehospitalario de la paciente con hemorragia obstétrica debe realizarse con un equipo multidisciplinario con conocimiento del uso de medicamentos uterotónicos y hemostásicos ${ }^{17}$.

Es importante mencionar también que la seguridad del ATX en traumatismos craneoencefálicos sigue en debate. Algunos autores demostraron la correlación entre la administración tardía de ATX y el riesgo de complicaciones tromboembólicas, y se considera que su administración durante las primeras $3 \mathrm{~h}$ después del trauma es la más segura ${ }^{18}$. Particularmente, aún existe controversia sobre su uso en sangrado craneal ${ }^{19}$.

Recientemente fue incluido en la lista de medicamentos esenciales de la Organización Mundial de la Salud $(\mathrm{OMS})^{20}$ y ha tenido validación clínica en varios estudios, incluyendo el Clinical Randomization of an Antifibrinolytic in Significant Hemorrhage (CRASH-2) y el Military Applicaction of Tranexamic Acid in Trauma Emergency Resucitation Study (MATTERS) ${ }^{21,22}$. En la literatura sobre atención médica prehospitalaria, como el Advanced Trauma Life Support (ATLS) ${ }^{21}$ y el PreHospital Trauma Life Support (PHTLS) ${ }^{23}$, se recomienda el uso del ATX en pacientes con hemorragias severas, sustentado en las tasas de éxito que ha tenido este medicamento ${ }^{22}$.

El ácido aminocaproico y el ATX actúan como potentes inhibidores de la fibrinólisis. Su acción biológica se basa en su unión competitiva a los lugares de unión de la lisina en el coágulo de fibrina, compitiendo de esta forma con el ligando del plasminógeno. La alteración de la unión del plasminógeno a la fibrina retrasa la conversión del plasminógeno a plasmina y la subsecuente fibrinólisis mediada por esta. Debido a que la potencia biológica del segundo es superior en unas 10 veces a la del ácido aminocaproico, la mayor parte de los trabajos se han centrado en el ATX (Tabla 1) ${ }^{24}$.

El objetivo principal de esta revisión es considerar la viabilidad del uso del ATX en el ámbito prehospitalario en México.

\section{Importancia del ácido tranexámico en el contexto prehospitalario}

En años recientes se ha estudiado considerablemente el influjo de la coagulopatía en el agravamiento de las hemorragias producidas en pacientes con trauma ${ }^{8}$. La solución se ha enfocado en el uso de antifibrinolíticos como una de las estrategias claves para mitigar las consecuencias fisiológicas de la hemorragia. De esta forma, el paradigma de la selección de prioridades en el tratamiento de los pacientes con trauma ha sido discutido ampliamente: ha pasado del simple control de daños a la más compleja reanimación homeostásica del paciente ${ }^{23}$. Así, lejos de concentrarse en grandes volúmenes de soluciones cristaloides para recuperar la tensión arterial normal, se están atendiendo temas más controvertidos, como mantener la perfusión mínima aceptable, facilitar la formación de coágulos y evitar los problemas asociados a la coagulopatía $a^{23,25,26 .}$.

El ATX se ha convertido en un antifibrinolítico ampliamente estudiado. Si bien es cierto que los ensayos clínicos que lo han utilizado tienen algunas limitaciones, como la falta de información recopilada o la cantidad de pacientes atendidos ${ }^{22}$, se ha observado en su mayoría disminución de la mortalidad de los pacientes en las primeras 24 horas. Es importante destacar que los ensayos clínicos comprenden un conocimiento y aplicación completos de ATLS, PHTLS, soporte vital avanzado, traslado rápido y pronta aplicación de ATX, es decir, el tratamiento prehospitalario del estado hipovolémico del paciente muestra mejores resultados 


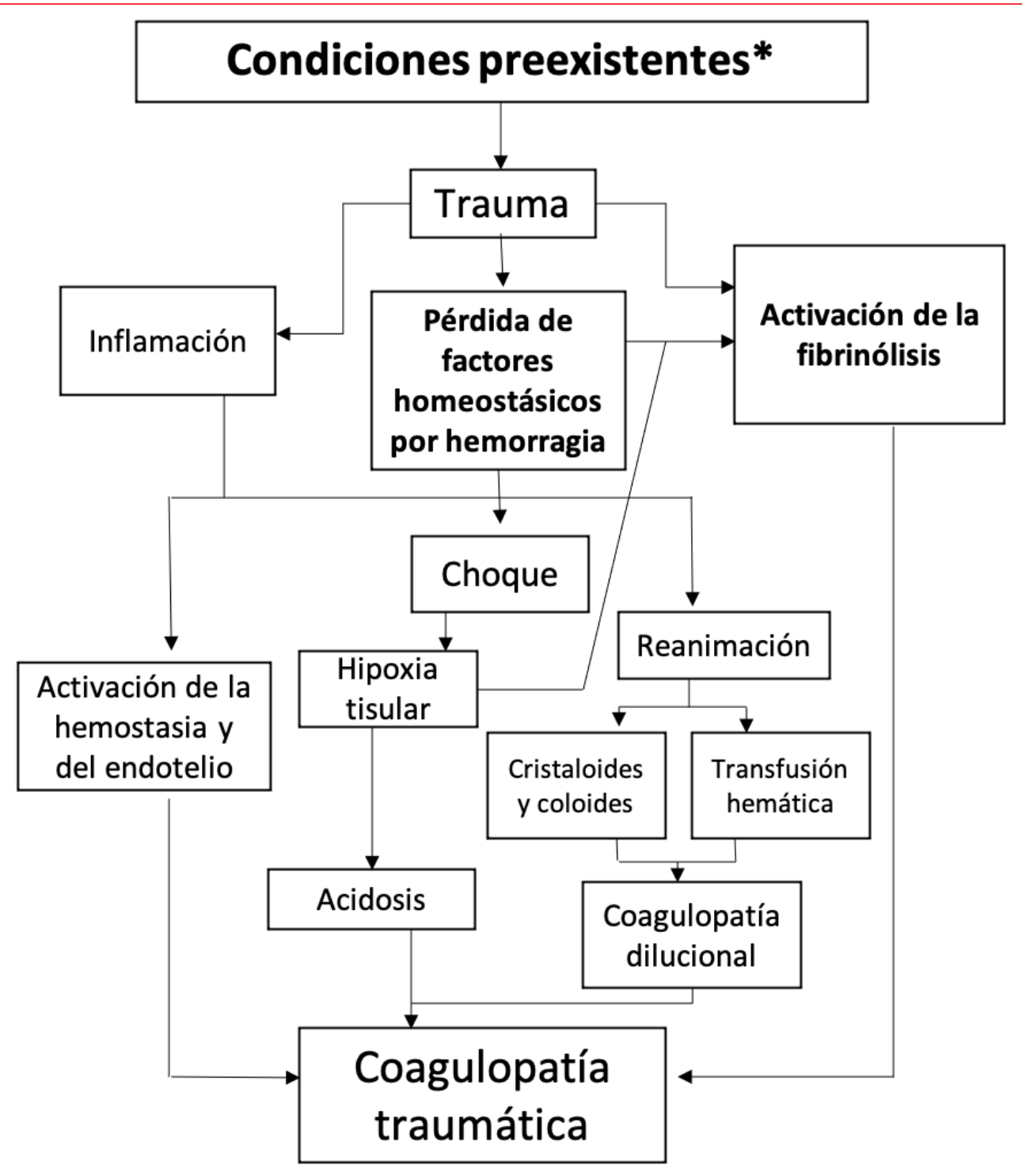

Figura 1. Conceptos de patogénesis de la coagulopatía posterior a trauma (adaptada de Soto-Estrada, et al. ${ }^{1}$ y Fernández-Lara, et al.'2).

*Referente a alteraciones genéticas, antecedentes patológicos y medicamentos, en especial los antitrombóticos.

cuando el ATX se aplica antes del arribo a la institución hospitalaria ${ }^{22}$. Incluso en los protocolos europeos, el ATX se sigue considerando como el único medicamento prehospitalario diseñado para apoyar la coagulación y se recomienda su administración ${ }^{27,28}$.

Existen también otros estudios prehospitalarios que evalúan la dinámica de la formación del coágulo y la degradación usando tromboelastometría (similar a la tromboelastografía). Se encontró que la capacidad del paciente para mantener la coagulación mejora significativamente después de la administración de ATX en el ambiente prehospitalario ${ }^{29}$.

\section{El ácido tranexámico en ensayos clínicos prehospitalarios}

Los protocolos más conocidos sobre el uso de ATX son el CRASH-2 y el MATTERS. El primero es el estudio más referenciado sobre el ATX y la homeostasis. Este ensayo clínico se realizó en 20,211 pacientes en 40 países, donde se observó que el riesgo de mortalidad disminuyó en un 1.5\% y la muerte causada por choque hipovolémico se redujo en un $0.8 \%$; todos los pacientes recibieron ATX en una dosis de 1 gramo inicial y 1 gramo en las siguientes horas ${ }^{30,31}$. El segundo 
Tabla 1. Comparación farmacológica entre el ácido tranexámico y el ácido aminocaproico

\begin{tabular}{|c|c|c|}
\hline & Ácido tranexámico & Ácido aminocaproico \\
\hline $\begin{array}{l}\text { Mecanismo } \\
\text { de acción }\end{array}$ & $\begin{array}{l}\text { - Forma un complejo } \\
\text { reversible que } \\
\text { desplaza el } \\
\text { plasminógeno de la } \\
\text { fibrina, dando lugar } \\
\text { a la inhibición de la } \\
\text { fibrinólisis } \\
\text { - Inhibe la actividad } \\
\text { proteolítica de la } \\
\text { plasmina } \\
\text { - Reduce la activación } \\
\text { del complemento y } \\
\text { el consumo del } \\
\text { inhibidor de la } \\
\text { esterasa } \\
\text { C1 (C1-INH), } \\
\text { disminuyendo la } \\
\text { inflamación }\end{array}$ & $\begin{array}{l}\text { - Se une } \\
\text { competitivamente al } \\
\text { plasminógeno } \\
\text { - Bloquea la unión } \\
\text { del plasminógeno a } \\
\text { la fibrina y la } \\
\text { posterior } \\
\text { conversión a } \\
\text { plasmina, dando } \\
\text { como resultado la } \\
\text { fibrinólisis }\end{array}$ \\
\hline $\mathrm{Vd}$ & 9 a 12 I & Oral: 23 I; IV: 30 I \\
\hline $\begin{array}{l}\text { Unión a } \\
\text { proteínas }\end{array}$ & $\begin{array}{l}3 \% \text {, principalmente al } \\
\text { plasminógeno }\end{array}$ & Mínima \\
\hline Metabolismo & $\begin{array}{l}\text { Mínimamente } \\
\text { hepático }\end{array}$ & $\begin{array}{l}\text { Mínimamente } \\
\text { hepático }\end{array}$ \\
\hline $\begin{array}{l}\text { Eliminación } \\
\text { de vida } \\
\text { media }\end{array}$ & 2 a $11 \mathrm{~h}$ & 1 a $2 \mathrm{~h}$ \\
\hline Excreción & Orina & Orina \\
\hline
\end{tabular}

Vd: volumen de distribución; IV: intravenoso.

ensayo clínico se realizó en un hospital militar estadounidense en Afganistán, en un lapso de dos años. Aquí se estudió la mortalidad a 24 y 48 horas, así como a 30 días. Se encontró que la mortalidad disminuyó del 18.9 al $11.3 \%$ a las primeras 48 horas. La mortalidad general en el MATTERS se redujo del 23.9 al $17.4 \%$ con el uso de ATX y por lo menos un paquete globular ${ }^{22}$. Ambos ensayos clínicos muestran el potencial beneficio del uso del ATX en el ámbito prehospitalario.

Si bien esos dos ensayos clínicos han sido los más relevantes para la comunidad médica, existe más evidencia que obtuvo resultados similares. A continuación analizaremos los estudios realizados por el General German Automobile Club (ADAC) Air Rescue Service y el California Prehospital Antifibrinolytic Therapy (CalPat). En estos, además del CRASH-2 y MATTERS, los criterios clínicos generales de inclusión de los pacientes estaban relacionados con la inestabilidad hemodinámica producida por trauma con una hemorragia severa: hipotensión, taquicardia o si se sospechaba choque hipovolémico compensado ${ }^{31}$.
En el ensayo clínico del ADAC, realizado con dos grupos de 258 pacientes cada uno, se observó una disminución en la mortalidad a 24 horas del 12.4 al $5.8 \%$, mientras que la mortalidad general disminuyó del 16.3 al $14.7 \%$. Este estudio, realizado en un lapso de dos años, indica de forma general la administración de 1 gramo de ATX a los pacientes, aunque la dosis específica de cada caso no está documentada ${ }^{8}$. En el estudio Cal-Pat, realizado en un lapso de 9 meses con 128 y 125 pacientes en el grupo de ATX y el de control respectivamente, también se advirtió una disminución en la mortalidad a 24 horas. La diferencia de esa tasa fue del 7.2 vs. $3.9 \%$ del grupo que recibió el fármaco, en una dosis de 1 gramo sobre 100 mililitros de solución salina al $0.9 \%$ en 10 minutos $^{32}$.

La evidencia presentada en estos cuatro estudios sugiere que la administración de ATX puede ser plausible por personal prehospitalario. Sin embargo, es fundamental destacar que en todos los ensayos clínicos se hace especial énfasis en el adecuado tratamiento del trauma descrito por protocolos como el ATLS, donde se da gran importancia a la detección temprana de choque hemorrágico, traslado rápido y prevención de pérdida de calor de los pacientes, entre otras medidas estandarizadas ${ }^{21,23,33}$.

\section{Discusión}

La premisa que motivó este artículo de revisión es que en México una de las principales causas de muerte en la población joven es el choque hemorrágico asociado a trauma ${ }^{1}$. Cada vez existe más evidencia de la seguridad en el uso del ATX en distintas áreas de la medicina. El tratamiento oportuno del choque hemorrágico de los pacientes se asocia directamente con una disminución en la mortalidad y mejor pronóstico. Por esta razón, consideramos que el uso del ATX en el ámbito prehospitalario es una opción que podría ser fácilmente implementada de acuerdo con la indicación y supervisión del médico del centro regulador para ajuste de dosis, vía de administración, evaluación de respuesta al tratamiento y seguimiento ${ }^{17}$.

En el contexto de trauma, la dosis sugerida actualmente es 1 gramo en 10 minutos, seguido de una infusión de 1 gramo en 8 horas. En los estudios realizados se ha demostrado que la disminución de la morbimortalidad es más significativa cuando este fármaco se administra en las primeras 3 horas posteriores al trauma ${ }^{34}$. Por tanto, resulta vital su apremiante administración. De esta forma, diversos países han implementado la aplicación prehospitalaria de la primera dosis, con el propósito de tener 
un beneficio real en los pacientes con choque hemorrágico. Los pacientes que se benefician con este manejo son aquellos con trauma y sangrado o bien aquellos con riesgo significativo de hemorragia.

Resulta importante mencionar que el ATX también cuenta con presentación vía oral con diferentes aplicaciones, el cual no interfiere con los protocolos de ayuno, según la Asociación Americana de Anestesiología ${ }^{35}$. Este medicamento cuenta con pocas contraindicaciones absolutas y no se requieren condiciones especiales para su almacenamiento y transporte. Incluso se ha comprobado su eficacia en la administración tópi$\mathrm{ca}^{36}$. Sin embargo, para el paciente crítico solo se ha demostrado su eficacia vía intravenosa, como lo indican los protocolos actuales.

Actualmente no se cuenta con estadísticas sobre el costo de la hemorragia crítica en México, pero considerando los datos de otros países, consideramos que la inversión podría disminuir con el uso de ATX en este tipo de pacientes. Su implementación también podría resultar factible debido a que no se requiere capacitación adicional para su manejo y administración por el rango de dosis terapéutica que tiene este medicamento. Actualmente no se describe su uso en los protocolos de los sistemas prehospitalarios más importantes en México, pese a que cuenta con validación sanitaria de la Comisión Federal para la Protección contra Riesgos Sanitarios (Cofepris) desde el 2011 ${ }^{37}$.

Si bien es cierto que no se cuenta con datos específicos sobre el costo de este medicamento en México, un estudio que compara los beneficios del ATX en países de bajo, medio y alto ingreso nos permite hacer algunas deducciones ${ }^{31}$. Incluso, la evidencia de su relación costo-efectividad fue un factor decisivo para incluirlo en la lista de medicamentos esenciales de la OMS ${ }^{15}$. Así, en países con escasez de sangre, el ATX puede reducir la mortalidad, como se ha comentado previamente; mientras que en países sin problemas en los bancos de sangre, el ATX puede reducir el número de infecciones y costos relacionados con las transfusiones ${ }^{38}$.

Un análisis estadístico de la evidencia del CRASH-2 en Tanzania, India e Inglaterra demuestra que para países de bajo, medio y alto ingreso (según datos del Banco Mundial con relación al producto interno bruto) $)^{39}$ es costo-efectivo para el sector salud invertir en ATX. Este estudio utilizó un modelo de Markov y una función genérica de supervivencia de Gompertz como herramientas estadísticas para medir la costo-efectividad en dólares por número de años ganados (LY, por sus siglas en inglés), con cual se encontró que la administración temprana de ATX podría salvar un estimado de
372, 315 y 755 LY en Tanzania, India e Inglaterra respectivamente. Así, se calcula que la administración prehospitalaria de ATX podría salvar entre 70,000 y 100,000 vidas por año. Esto se debe, por un lado, a que más del $90 \%$ de las muertes son consecuencia del trauma en países con bajo y medio ingreso. Así, el potencial el ATX para salvar vidas en esos contextos específicos es particularmente alta. Por otro lado, en países de alto ingreso, los LY se traducen en productividad de los pacientes, por lo tanto se presume que generaría un potencial crecimiento económico ${ }^{31}$.

De esta forma, se ha analizado la evidencia existente y se ha encontrado que el uso del ATX en el contexto prehospitalario es benéfico para los pacientes. Esto se apoya en la cantidad de estudios y protocolos que lo sustentan, además de ser un producto costo-eficiente para los países sin importar su nivel de ingreso. Así, consideramos que el tratamiento basado en administrar grandes cantidades de soluciones no es tan efectivo como el control de la coagulopatía. Por tanto, este fármaco debería incluirse en los protocolos mexicanos de atención prehospitalaria, además de emplearse de manera oportuna, acompañado del tratamiento adecuado del paciente de trauma.

\section{Conclusiones}

- Existe un cambio de paradigma, el cual ha replanteado el tratamiento de la hemorragia crítica en los pacientes.

- Este artículo pretende estudiar el posible efecto en los pacientes de trauma iniciando desde una perspectiva prehospitalaria, aunque también es fundamental hacer énfasis en que la incipiente exploración de este tipo de tratamientos ha resultado exitosa y ha atraído la atención de la comunidad médica.

- El momento más adecuado para comenzar el tratamiento de los pacientes con choque hipovolémico por hemorragia es en el periodo prehospitalario.

- El ATX es un medicamento seguro que disminuye la cantidad de sangrado en los pacientes, disminuyendo la mortalidad y evitando complicaciones.

- Basándonos en toda la evidencia disponible y en la literatura de múltiples disciplinas, el ATX es seguro y costo-eficiente para el uso prehospitalario en México.

\section{Financiamiento}

La presente investigación no ha recibido ninguna beca específica de agencias de los sectores públicos, comercial, o sin ánimo de lucro. 


\section{Conflicto de intereses}

Los autores declaran no tener conflicto de intereses alguno.

\section{Responsabilidades éticas}

Protección de personas y animales. Los autores declaran que para esta investigación no se han realizado experimentos en seres humanos ni en animales.

Confidencialidad de los datos. Los autores declaran que en este artículo no aparecen datos de pacientes.

Derecho a la privacidad y consentimiento informado. Los autores declaran que en este artículo no aparecen datos de pacientes.

\section{Bibliografía}

1. Soto-Estrada G, Moreno-Altamirano L, Pahua Díaz D. Panorama epidemiológico de México, principales causas de morbilidad y mortalidad. Rev Fac Med (Mex). 2016;56(6):8-22.

2. Fernández-Lara JA, Toro-Ortiz JC, Martínez-Trejo Z, de la Maza-Labastida S, Villegas-Arias MA. Tasa de hemorragia, histerectomía obstétrica y muerte materna relacionada. Ginecol Obstet Mex. 2017;85(4):247-53.

3. Carrillo-Esper R, Peña-Pérez CA. Definiciones y abordaje de la hemorragia crítica. Rev Mex Anestesiol. 2015;38(2):S374-S379.

4. Prevención y manejo de la hemorragia postparto en primero, segundo y tercer niveles de atención. Evidencias y Recomendaciones. Guía de Práctica Clínica [Internet]. México: Secretaría de Salud, Centro Naciona de Excelencia Tecnológica; 2017. Disponible en: http://www. cenetec-difusion.com/CMGPC/SS-103-08/ER.pdf

5. Watts G. Obituary. Utako Okamoto. Lancet. 2016;10035(387):2286

6. Sidelmann JJ, Gram J, Jespersen J, Kluft C. Fibrin clot formation and lysis: Basic mechanisms. Semin Thromb Hemost. 2000;26(6):605-18.

7. $\mathrm{Ng} \mathrm{W}$, Jerath $\mathrm{A}$, Wasowicz M. Tranexamic acid: a clinical review. Anaesthesiol Intensive Ther. 2015;47(4):339-50.

8. Wafaisade A, Lefering R, Bouillon B, Böhmer AB, Gäßler M, Ruppert M TraumaRegister DGU. Prehospital administration of tranexamic acid in trauma patients. Crit Care. 2016;143(20):1-9.

9. Kashuk JL, Moore EE, Sawyer M, Wohlauer M, Pezold M, Barnett C et al. Primary fibrinolysis is integral in the pathogenesis of the acute coagulopathy of trauma. Ann Surg. 2010;252(3):434-42.

10. Spahn DR, Bouillon B, Cerny V, Coats TJ, Duranteau J, FernándezMondéjar $\mathrm{E}$, et al. Management of bleeding and coagulopathy following major trauma: An updated European guideline. Crit Care. 2013;17(2):R76.

11. García-Chávez J, Carrillo-Esper R, Majluf-Cruz A. Fisiología del sistema de coagulación. Gac Med Mex. 2007;143(1):7-9

12. Roberts I, Coats T, Edwards P, Gilmore I, Jairath V, Ker K, et al. HALT-IT - tranexamic acid for the treatment of gastrointestinal bleeding: study protocol for a randomised controlled trial. Trials. 2015;450(14):1-14.

13. Mi B, Liu G, Zhou W, Lv H, Liu Y, Zha K, et al. Intra-articular versus intravenous tranexamic acid application in total knee arthroplasty: A meta-analysis of randomized controlled trials. Arch Orthop Trauma Surg. 2017:137(7)997-1009

14. Van Aelbrouck C, Jorquera-Vasquez S, Beukinga I, Pradier O, Ickx B Barvais $L$, et al. Tranexamic acid decreases the magnitude of platelet dysfunction in aspirin-free patients undergoing cardiac surgery with cardiopulmonary bypass. Blood Coagul Fibrinolysis. 2016;27(8):855-61.

15. Windyga J, Stefanska-Windyga E, Odnoczko E, Baran B, Czubak G. Activated prothrombin complex concentrate in combination with tranexamic acid: a single centre experience for the treatment of mucosal bleeding and dental extraction in haemophilia patients with inhibitors. Haemophilia. 2016;22:e435-e493.

16. Eckert MJ, Wertin TM, Tyner SD, Nelson DW, Izenberg S, Martin MJ. Tranexamic acid administration to pediatric trauma patients in a combat setting: The pediatric trauma and tranexamic acid study (PED-TRAX). J Trauma Acute Care Surg. 2014;77(6):852-8.

17. WOMAN Trial Collaborators. Effect of early tranexamic acid administration on mortality, hysterectomy, and other morbidities in women with post-partum haemorrhage (WOMAN): An international, randomised, double-blind, placebo-controlled trial. Lancet. 2017;389(10084):2105-16.
18. Chakroun-Walha O, Samet A, Jerbi M, Nasri A, Talbi A, Kanoun H, et al. Benefits of the tranexamic acid in head trauma with no extracranial bleeding: a prospective follow-up of 180 patients. Eur J Trauma Emerg Surg. 2019;45:719-26.

19. Fakharian $\mathrm{E}$, Abedzadeh-kalahroudi $\mathrm{M}$, Atoof $\mathrm{F}$. Effect of tranexamic acid on prevention of hemorrhagic mass growth in patients with traumatic brain injury. World Neurosurg. 2018;109:e748-e753.

20. Carrillo-Esper R, de la Torre-León T, Nava-López JA, Posada-Nava A Pérez-Calatayud AA, de la Torre-León MA, et al. Consenso multidisciplinario para el manejo de la hemorragia obstétrica en el perioperatorio. Rev Mex Anestesiol. 2018;41(3):155-82.

21. World Health Organization. The selection and use of essential medicines. Report of the WHO Expert Committee, 2017 (including the 20th WHO Model List of Essential Medicines and the 6th Model List of Essential Medicines for Children) [Internet]. World Health Organization; 2017. Disponible en: https://apps.who.int/iris/handle/10665/259481? locale-attribute=es\&

22. American College of Surgeons. ATLS. Advanced trauma life support. Student Course Manual. Tenth edition. EE.UU.: American College of Surgeons; 2018.

23. National Association of Emergency Medical Technicians. PHTLS. Prehospital Trauma Life Support. 8th edition. EE.UU.: National Association of Emergency Medical Technicians; 2014.

24. Quintana Díaz M, Cabestrero Alonso D, García de Lorenzo y Mateos A. Coagulación y hemorragia en el paciente crítico. Parte II. Factor pronóstico y tratamiento. Med Intensiva. 2003;27(10):676-85.

25. Vu EN, Schlamp RS, Wand RT, Kleine-Deters GA, Vu MP, Tallon JM Prehospital use of tranexamic acid for hemorrhagic shock in primary and secondary air medical evacuation. Air Med J. 2013;32(5):289-92.

26. Ausset S, Glassberg E, Nadler R, Sunde G, Cap AP, Hoffmann C, et al. Tranexamic acid as part of remote damage-control resuscitation in the prehospital setting: A critical appraisal of the medical literature and available alternatives. J Trauma Acute Care Surg. 2015;78(6):S70-S75.

27. Spahn DR, Bouillon B, Cerny V, Coats TJ, Duranteau J, FernándezMondéjar $E$, et al. Management of bleeding and coagulopathy following major trauma: An updated European guideline. Crit Care. 2013:17(2):R76.

28. Stein $P$, Studt JD, Albrecht R, Müller S, von Ow D, Fischer S, et al. The impact of prehospital tranexamic acid on blood coagulation in trauma patients. Anesth Analg. 2018;126(2):522-9.

29. Rossaint R, Bouillon B, Cerny V, Coats TJ, Duranteau J, Fernández-Mondéjar $\mathrm{E}$, et al. The European guideline on management of major bleeding and coagulopathy following trauma: fourth edition. Crit Care. 2016;20:100.

30. Kunze-Szikszay N, Krack LA, Wildenauer $P$, Wand S, Heyne $T$, Walliser $\mathrm{K}$, et al. The prehospital administration of tranexamic acid to patients with multiple injuries and its effects on rotational thrombelastometry: A prospective observational study in prehospital emergency medicine. Scand J Trauma Resusc Emerg Med. 2016;24(1):122.

31. Fischer PE, Bulger EM, Perina DG, Delbridge TR, Gestring ML, Fallat ME, et al. Guidance document for the prehospital use of tranexamic acid in injured patients. Prehosp Emerg Care. 2016;20(5):557-9.

32. Guerriero C, Cairns J, Perel P, Shakur H, Roberts I; CRASH 2 collaborators. Cost-effectiveness analysis of administering tranexamic acid to bleeding trauma patients using evidence from the CRASH-2 Trial. PLoS One. 2011;6(5):e18987.

33. Carr MJ, DiCorpo JE, Mell HK, Merlin MA, Weingart SD. An interdisciplinary literature review of prehospital use of tranexamic acid (TXA) in major bleeding [Internet]. San Antonio, TX: JEMS; 01/01/2018. Disponible en: https://www.jems.com/patient-care/an-interdisciplinary-literature-review-of-prehospital-use -of-tranexamic-acid-txa-in-major-bleeding/.

34. Neeki MM, Dong F, Toy J, Vaezazizi R, Powell J, Jabourian N, et al. Efficacy and safety of tranexamic acid in prehospital traumatic hemorrhagic shock: Outcomes of the Cal-PAT Study. West J Emerg Med. 2017;18(4):673-83

35. Strosberg DS, Nguyen MC, Mostafavilar L, Mell H, Evans DC Development of a prehospital tranexamic acid administration protocol. Prehosp Emerg Care. 2016;20(4):462-6.

36. American Society of Anesthesiologists. Practice guidelines for preoperative fasting and the use of pharmacologic agents to reduce the risk of pulmonary aspiration: Application to healthy patients undergoing elective procedures. Anesthesiology. 2017;126(3):376-93.

37. Zahed R, Jazayeri MHM, Naderi A, Naderpour Z, Saeedi M. Topical tranexamic acid compared with anterior nasal packing for treatment of epistaxis in patients taking antiplatelet drugs: Randomized controlled trial. Acad Emerg Med. 2018;25(3):261-6.

38. Comisión Federal para la protección contra Riesgos Sanitarios. México: Comisión de Autorización Sanitaria, Dirección Ejecutiva de Autorización de Productos y Establecimientos Registros de Medicamentos Prorrogados en 2017. Disponible en: www.innsz.mx/2017/ Noticias/ Sintesis08mayo2017.pdf

39. Guerriero C, Cairns J, Jayaraman S, Roberts I, Perel P, Shakur H. Giving tranexamic acid to reduce surgical bleeding in sub-Saharan Africa: an economic evaluation. Cost Eff Resour Alloc. 2010;8(1):1. 